\title{
SHUYU Robot: An Automatic Rapid Temperature Screening System
}

\author{
Zhao Gong ${ }^{1}$, Songwen Jiang ${ }^{1}$, Qizhi Meng ${ }^{1}$, Yanlei Ye ${ }^{1}$, Peng Li ${ }^{1}$, Fugui Xie ${ }^{1,2}$, Huichan Zhao ${ }^{1,2}$, Chunzhe Lv $^{3}$, \\ Xiaojie Wang ${ }^{4}$ and Xinjun Liu ${ }^{1,2^{*}}$
}

The health of people around the world and the global economy are under substantial threat from the outbreak of pandemics [1]. Controlling pandemics is extremely challenging, with preventing the spread of pathogens the most important and critical step. Of all preventative actions, body temperature screening is undoubtedly highly necessary and effective [2].

During an infectious disease pandemic, automobile passengers and pedestrians should be manually screened at all types of entrances, including those to highways, airports, bus stations, hospitals, schools, conference venues, exhibition halls, office areas, hotels, and supermarket parking lots. Screening tasks are currently conducted by security guards or other staff via infrared (IR) thermometers.

Currently, two types of thermometer are widely used for temperature measurement, i.e., contact and noncontact thermometers [3]. Contact-based measurements are usually time-consuming and increase the risk of cross infection between the humans being tested. In comparison, non-contact measurements have obvious advantages. Non-contact thermometers include IR screening instruments, IR forehead thermometers, and IR ear thermometers. Among them, forehead thermometers and screening instruments are most widely used, with typical accuracy levels of $0.5{ }^{\circ} \mathrm{C}$ and a maximum accuracy of $0.3^{\circ} \mathrm{C}$.

\footnotetext{
*Correspondence: xinjunliu@mail.tsinghua.edu.cn

${ }^{1}$ The State Key Laboratory of Tribology \& Tsinghua University

(DME)-Siemens Joint Research Center for Advanced Robotics,

Department of Mechanical Engineering (DME), Tsinghua University,

Beijing 100084, China

Full list of author information is available at the end of the article
}

In practice, the following problems are encountered when using forehead thermometers or screening instruments. 1) To achieve the expected accuracy, the ambient temperature is strictly limited (to approximately $20{ }^{\circ} \mathrm{C}$ ) and a wind-free environment is usually required, i.e., the default service environment is generally indoors. 2) Indoor use raises the possibility of bringing pathogens indoors, which increases the risk of cross infection and hinders pandemic control. 3) When measuring facial temperature, facial coverings (cosmetics, dust, hair, etc.) easily lead to inaccurate results. To avoid these problems, the only reasonable approach is to measure body temperature outdoors on skin covered by clothes.

As there is no available and reliable equipment for outdoor temperature measurement, forehead thermometers must be used outdoors. As a result, large deviations in temperature measurements are inevitable. In addition, temperature measurements by hand-held forehead thermometers are inefficient and highly labor intensive. Furthermore, the procedure might cause personal discomfort, and the possibility of cross infection still exists even outdoors. Therefore, a method for performing automatic rapid and accurate temperature measurements in an outdoor environment is urgently required.

In this context, the Tsinghua Advanced Mechanism and Robotized Equipment Laboratory and the Yantai Tsingke+ Robot Joint Research Institute Co., Ltd. have jointly developed an automatic rapid temperature screening system that is particularly suitable for outdoor environments. The research and development of this system are mainly focused on the robotic design, temperature measurement model design, compensation algorithm development, visual semantic perception, and control strategy. 
The automatic rapid temperature screening system has two different configurations. One is for drivers and passengers of mobile vehicles and the other is for pedestrians. The system has the following characteristics:

1. Semantic recognition and instance segmentation are achieved with a deep neural network. Using dual IR cameras, the number and position of passengers in the car are identified. The facial recognition rate exceeds $98 \%$ when the confidence is set to $92 \%$.

2. A constant-temperature measurement model is designed and an intelligent temperature compensation algorithm is established. On these bases, the accuracy of temperature measurement reaches $0.2^{\circ} \mathrm{C}$.

3. Multiple sensors are integrated with functions of visual recognition, ultrasonic detection or IR detection. Consequently, rapid positioning of vehicles, and automatic temperature screening of drivers, passengers, and pedestrians are realized. The detection time for a vehicle is less than $20 \mathrm{~s}$ and that for a passenger is less than $2 \mathrm{~s}$.

4. Using big-data technology, maps of vehicle, passenger, and temperature data are generated and uploaded to the cloud for management.

A schematic of the automatic rapid temperature screening system for drivers and passengers in mobile vehicles is presented in Figure 1. The system consists of two robots (named SHUYU robots). As shown in Figure 2, the SHUYU robot is composed of a two-degreesof-freedom translational parallel manipulator with a

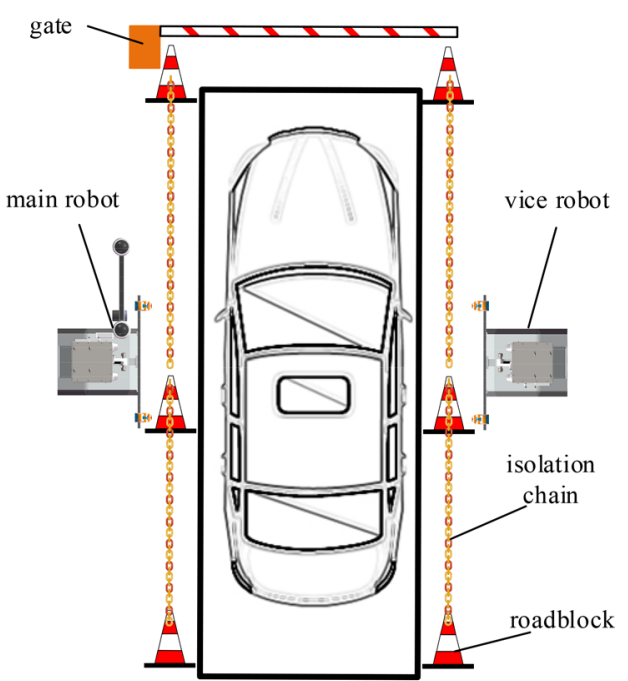

Figure 1 Schematic of the automatic rapid temperature screening system

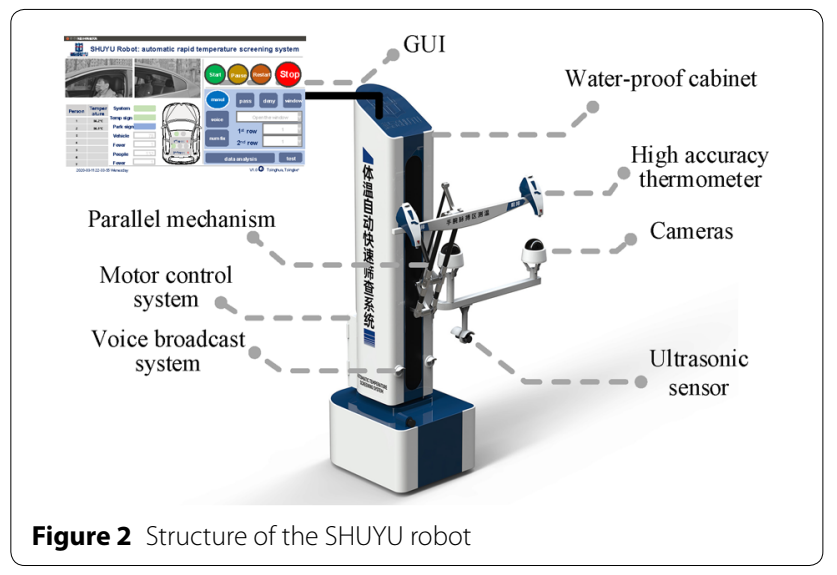

closed-loop passive limb [4], which can be represented by $\underline{\mathrm{P} P a}-\underline{\mathrm{P} R}$ ( $\underline{\mathrm{P}}$ : actuated prismatic joint; Pa: parallelogram mechanism; R: revolute joint).

Two high-accuracy thermometers developed for outdoor applications are installed at the end of each parallel manipulator. The main robot shown in Figure 1 is also equipped with two cameras (Figure 2) to monitor the driver and passengers. Both SHUYU robots are equipped with ultrasonic sensors to detect the distance between the robot and the vehicle. In addition, the robot is equipped with a voice broadcast system for guiding the passengers through the temperature measurement step by step. A total of four high-accuracy thermometers are included in the screening system; therefore, four passengers (including the driver) can be measured simultaneously. When the number of people in a vehicle exceeds four, a nearby thermometer can subsequently be used to complete the additional measurements. If all temperatures are within normal ranges, the gate in Figure 1 will open, allowing the vehicle to pass through; otherwise, the gate will remain closed and the system will sound an alarm.

To screen the temperatures of pedestrians at various entrances, the SHUYUmini robotic system presented in Figure 3 has been developed. The arm of SHUYUmini is a parallelogram mechanism with one degree of freedom. The high-accuracy thermometer is connected to the body of the robot through a damped spherical joint. The user can adjust the direction to find a comfortable measurement pose. Three laser-ranging sensors are arranged on the side of the robot to identify the position of pedestrians. Similarly, if the temperature is in a normal range, the arm will open; otherwise, the arm will remain closed and the system will sound an alarm.

To ensure accurate measurement, especially outdoors, the high-accuracy thermometer presented in Figure 4 has been developed. The main components are 

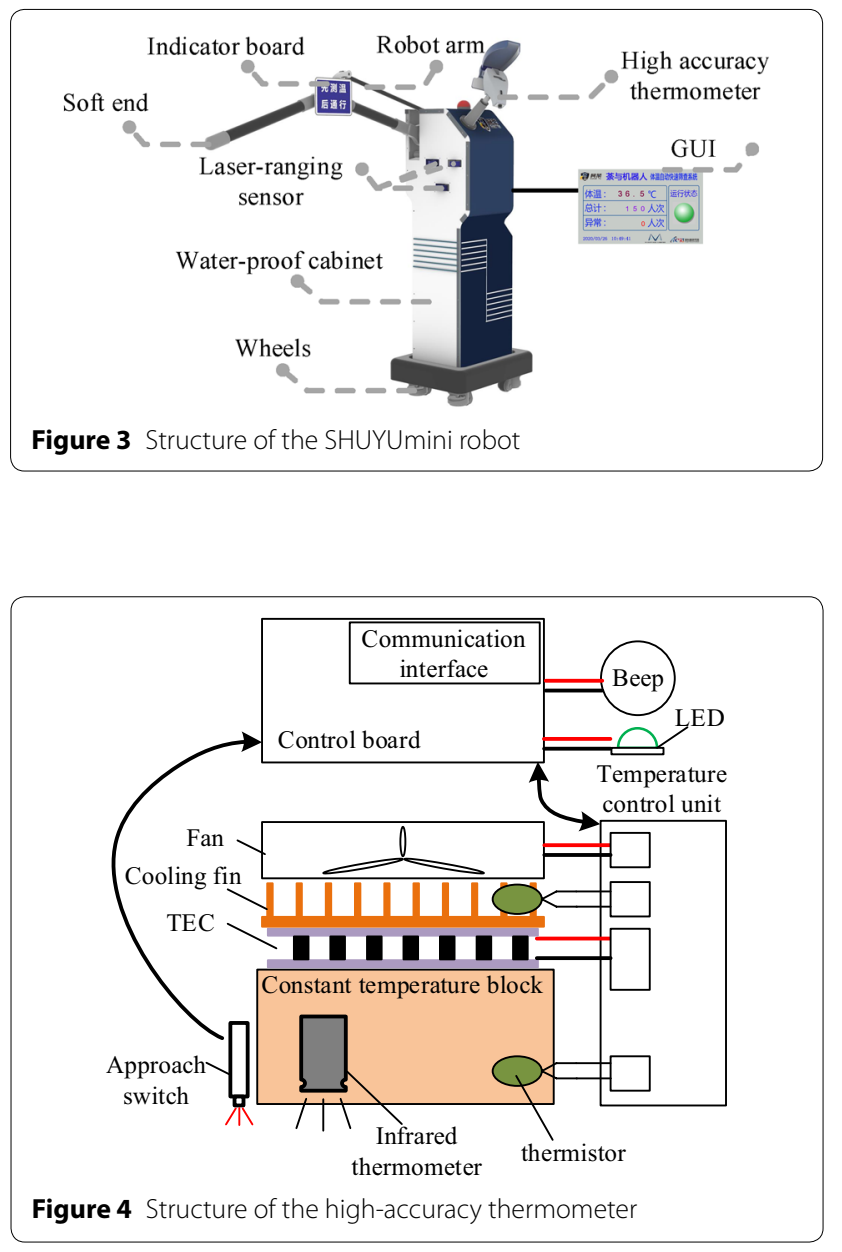

an IR thermometer, an approach switch, and a temperature control system. The temperature control system contains a thermo electric cooler (TEC), a fan and a cooling fin, two thermistors, and a temperature control unit. The IR thermometer is embedded in the constanttemperature block, and its temperature can be maintained at the desired value (approximately $20^{\circ} \mathrm{C}$ ) by the temperature control system. The approach switch can detect opaque objects within $3 \mathrm{~cm}$, allowing it to detect the approach of a wrist. A beep and LED are used to indicate when temperature data collection is finished.

The SHUYU robot control system mainly consists of two parts: a master unit and a slave unit. The overall framework of the control system is illustrated in Figure 5. The graphical user interface (GUI), the visual system, and the voice broadcast system are contained in the master unit. The master computer is responsible for processing the visual data and orders from the GUI; it then sends the necessary messages to the slave unit. The slave unit collects the distance and temperature data, then uploads these data to the master computer.

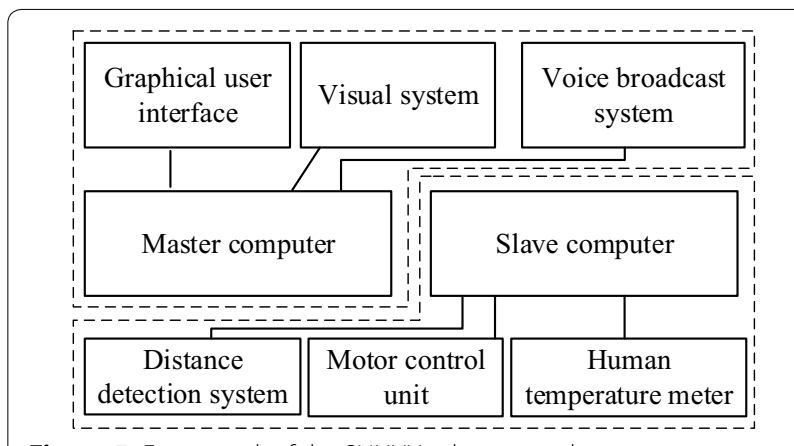

Figure 5 Framework of the SHUYU robot control system

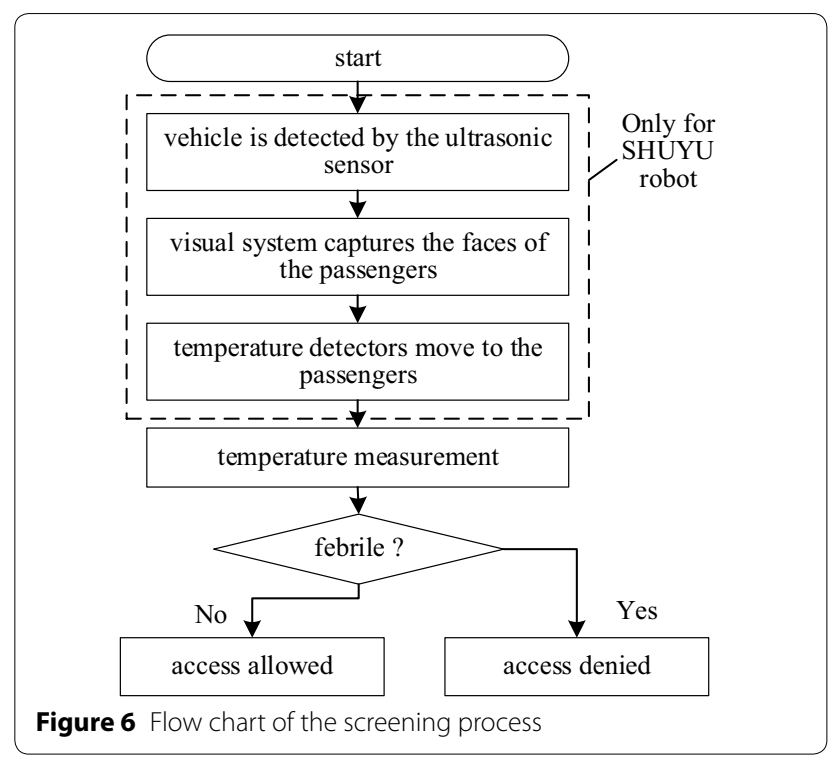

The motors are controlled directly by the slave unit but the orders are given by the master computer.

A flow chart of the screening process is presented in Figure 6. For temperature measurement of drivers and passengers in mobile vehicles, when the ultrasonic sensor of the SHUYU robot detects that a vehicle is approaching, the camera is triggered to visually identify the number and position of the driver and passengers. Then, the robot is controlled to extend the high-accuracy thermometers to an appropriate position. The driver and passengers then extend their wrists for temperature measurement, and the robot records the temperature data and determines whether the temperature qualifies the driver and passengers for access. For temperature measurement of pedestrians, the pedestrians extend their wrists for temperature measurement, and the SHUYUmini robot records the temperature data and determines whether the temperature qualifies the pedestrians for access. 


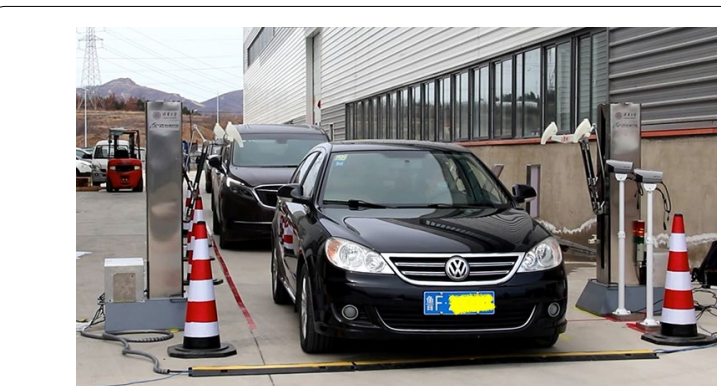

Figure 7 SHUYU robot used in Yantai ETDA

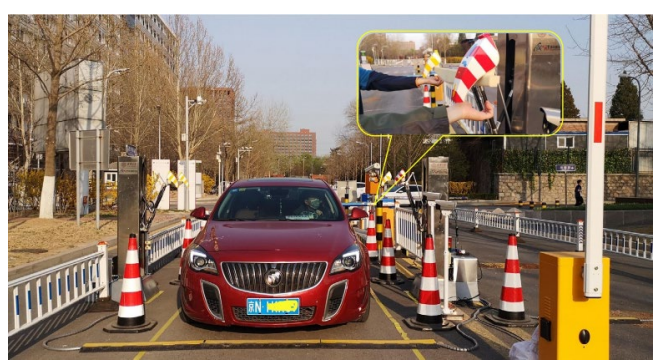

Figure 8 SHUYU robot used in Tsinghua University

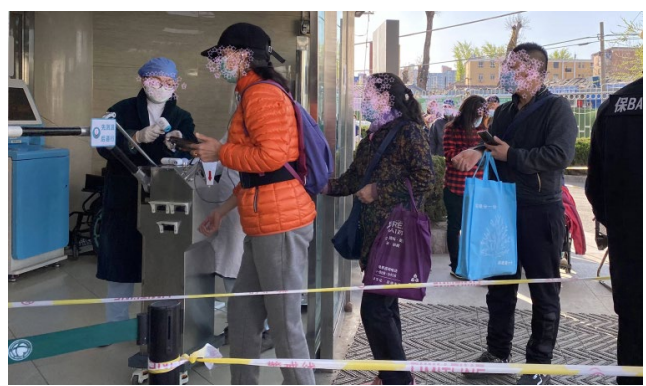

Figure 9 SHUYUmini robot used in the First Affiliated Hospital of Tsinghua University

Currently, the SHUYU screening system has been used in parking lots in Yantai ETDA (Figure 7) and Tsinghua University (Figure 8). The SHUYUmini screening system has been used in Peking Union Medical College Hospital, the First Affiliated Hospital of Tsinghua University (Figure 9), and the LeeShauKee Building of Tsinghua University.

The developed screening system can also be applied to highways, airports, bus stations, and checkpoints, as well as hospitals, schools, banks, conference venues, exhibition halls, office areas, hotels, residential quarters, supermarket parking lots, and any other channel requiring rapid automatic temperature screening.

\section{Authors' Contributions}

$\mathrm{XL}$ and $\mathrm{FX}$ were in charge of the whole trial; $\mathrm{QM}, \mathrm{FX}$, and $\mathrm{HZ}$ wrote the manuscript; ZG, SJ, YY, PL, CL, and XW assisted with sampling and laboratory analyses. All authors read and approved the final manuscript.

\section{Authors' Information}

Zhao Gong, born in 1990, is currently a Ph.D. candidate at Department of Mechanical Engineering (DME), Tsinghua University, China.

Songwen Jiang, born in 1995, is currently a Ph.D. candidate at DME, Tsinghua University, China.

Qizhi Meng, born in 1992, is currently a Ph.D. candidate at DME, Tsinghua University, China.

Yanlei Ye, born in 1991, is currently a Ph.D. candidate at DME, Tsinghua University, China.

Peng Li, born in 1989, is currently a Ph.D. candidate at DME, Tsinghua University, China.

Fugui Xie, born in 1982, is currently an associate professor at DME, Tsinghua University, China.

Huichan Zhao, born in 1989, is currently an assistant professor at DME, Tsinghua University, China.

Chunzhe Lv, born in 1980, is currently a senior engineer at Yantai Tsingke+ Robot Joint Research Institute Co., Ltd., China.

Xiaojie Wang, born in 1976, is currently an associate chief physician at The First Affiliated Hospital of Tsinghua University, China.

Xinjun Liu, born in 1971, is currently a professor and a Ph.D. candidate supervisor at DME, Tsinghua University, China. His research interests include robotics, parallel mechanisms, and advanced manufacturing equipment.

\section{Competing Interests}

The authors declare no competing financial interests.

\section{Author Details}

${ }^{1}$ The State Key Laboratory of Tribology \& Tsinghua University (DME)-Siemens Joint Research Center for Advanced Robotics, Department of Mechanical Engineering (DME), Tsinghua University, Beijing 100084, China. ${ }^{2}$ Beijing Key Lab of Precision/Ultra-precision Manufacturing Equipments and Control, Tsinghua University, Beijing 100084, China. ${ }^{3}$ Yantai Tsingke+ Robot Joint Research Institute Co., Ltd., Yantai 264000, Shandong, China. ${ }^{4}$ The First Affiliated Hospital of Tsinghua University, Beijing 100016, China.

Received: 13 April 2020 Accepted: 22 April 2020

Published online: 05 May 2020

\section{References}

[1] G-Z Yang, B J Nelson, R R Murphy, et al. Combating COVID-19-The role of robotics in managing public health and infectious diseases. Science Robotics, 2020, 5(40): eabb5589.

[2] E F J Ring. The historical development of temperature measurement in medicine. Infrared Physics \& Technology, 2007, 49(3): 297-301.

[3] D Bitar, A Goubar, J C Desenclos. International travels and fever screening during epidemics: a literature review on the effectiveness and potential use of non-contact infrared thermometers. Eurosurveillance, 2009, 14(6): 19115.

[4] Q Z Meng, F G Xie, X-J Liu, et al. An evaluation approach for motion-force interaction performance of parallel manipulators with closed-loop passive limbs. Mechanism and Machine Theory, 2020, 149: 103844. 